\title{
Schwannoma de colon: Reporte de caso y revisión de la literatura
}

\author{
Ricardo Vega F*, Andrés Vicentela I', Consuelo Gatica T', Gerhard Franz $G^{2}$, Matías Molina V. ${ }^{2}$
}

1. Residente de Radiología. Departamento de Imágenes, Facultad de Medicina, Clínica Alemana de Santiago - Universidad del Desarrollo. Santiago, Chile.

2. Radiólogo. Departamento de Imágenes, Hospital Padre Hurtado, Santiago, Chile.

\section{Colon schwannoma: Case report and literature review}

\begin{abstract}
Resumen:
Los schwannomas son neoplasias benignas que se originan de las células de Schwann que forman la vaina neural. El origen primario gastrointestinal es raro y corresponde a una entidad separada de los schwannomas convencionales que se originan en los tejidos blandos o en el sistema nervioso central. Cuando se presentan en el sistema gastrointestinal, la ubicación más habitual es en el estómago y con mucho menor frecuencia en colon. Se presenta el caso de un hombre de 74 años con una lesión colónica neoplásica en el ángulo hepático, asociado a un segmento corto invaginado y cambios inflamatorios adyacentes sometido a colonoscopía y posterior cirugía. La pieza quirúrgica demostró la presencia de cuerpos de Verocay y reactividad para los marcadores S-100 y GFAP respectivamente, lo cual confirmó la presencia de un schwanomma de colon.
\end{abstract}

Palabras clave: Inmunohistoquímica; Schwannoma de colon; Tomografía computada.

\begin{abstract}
:
Schwannomas are mostly benign neoplasms that arise from the Schwann cells that form the peripheral neural sheath. Schwannomas of the gastrointestinal tract are rare and are considered separate entities from conventional schwannomas that originate in the soft tissues or in the central nervous system. Most gastrointestinal schwannomas occur in the stomach. Colon schwannomas are quite rare. We present the case of a 74-year-old man who with a colonic neoplastic lesion on the hepatic flexure, associated with a short invaginated segment and inflammatory changes. He underwent colonoscopy and subsequent surgical resection. The surgical specimen showed the presence of Verocay bodies and reactivity to the S-100 and GFAP markers respectively, that confirmed the presence of a colon schwanomma.
\end{abstract}

Keywords: Colon schwannoma; Computed tomography; Immunohistochemistry.

Vega R., et al. Schwannoma de colon: Reporte de caso y revisión de la literatura. Rev Chil Radiol 2020; 26(4): 168-171. *Correo electrónico: Ricardo Vega F. / ricardovegafins@gmail.com

Trabajo enviado el 09 de mayo de 2020. Aceptado para publicación el 15 de agosto de 2020.

\section{Introducción}

Los schwannomas son tumores derivados de las células de Schwann que forman la vaina neural. Los que se originan del tracto gastrointestinal son tumores poco frecuentes y surgen a partir de las células de Schwann de los nervios periféricos, constituyendo el $2-6 \%$ de todos los tumores mesenquimales. Aparecen habitualmente en estómago y en segundo lugar en el colon, donde lo más frecuente es encontrarlos en ciego seguido del sigmoides ${ }^{1}$.

Los schwannomas primarios del colon (SC) suelen ser benignos, pero de forma inhabitual (2\%), pueden presentar degeneración maligna ${ }^{2}$. Suelen presentarse como un pólipo que puede ulcerar la 
mucosa dando síntomas inespecíficos como dolor abdominal con sangrado rectal, dificultad defecatoria, obstrucción colónica o invaginación ${ }^{3,4}$. Las pruebas de imagen son generalmente inespecíficas y pueden demostrar una masa mural bien definida y de realce homogéneo ${ }^{5}$. La mayoría de las veces la biopsia no es diagnóstica, obteniéndose un resultado adecuado solo con la obtención de la pieza quirúrgica completa ${ }^{6}$. Al estudio microscópico se disponen en un patrón de haces entrelazados, rodeada de un infiltrado de células linfoides con centros germinales y pequeños focos de atipia celular con escasa actividad mitótica? . Sin técnicas de inmunohistoquímica, estos tumores pueden presentar características similares a los leiomiomas o tumores del estroma gastrointestinal ${ }^{8}$.

Se presenta el caso de un SC confirmado mediante técnica inmunohistoquímica.

\section{Caso clínico}

Paciente masculino de 74 años, con antecedentes de hipertensión arterial, diabetes mellitus tipo 2 y apendicectomía. Consulta por baja de peso de aproximadamente $5 \mathrm{~kg}$ en 2 meses, asociado en las últimas dos semanas a hematoquezia y melena. Al examen físico solo destacaba a la palpación una masa supraumbilical. Se decide hospitalizar para continuar el estudio. Se realizaron exámenes de laboratorio entre los que destacan: Hb: 12.5 g/dl, Hto:
36.4\%, Leucocitos: $7700 / \mathrm{mm}^{3}$, plaquetas: 206.000 $\mathrm{mm}^{3}$, albumina: $3.8 \mathrm{~g} / \mathrm{dl}$, TP: $14.9 \mathrm{seg}$, TTPA: $28.6 \mathrm{seg}$ y INR: 1.24. Se solicita una tomografía computada (TC) de abdomen y pelvis con contraste, donde se evidenció una masa en el ángulo hepático, asociado a un segmento corto invaginado y marcados cambios inflamatorios de la grasa adyacente; la lesión presentaba marcado realce homogéneo en la fase porto-venosa (Figuras 1a y 1b). No se identificó extensión a órganos adyacentes ni presencia de diseminación a distancia.

Se decidió realizar una colonoscopia que evidenció una lesión polipoidea neoplásica, de consistencia dura y friable al tacto (Figura 2). Se obtuvieron múltiples biopsias incisionales con resultado de adenoma tubular de colon con displasia de bajo grado. Se decide la resolución con una hemicolectomia derecha abierta con anastomosis ileo-transverso término lateral, sin evidenciarse compromiso linfático, hepático ni peritoneal. La pieza quirúrgica fue enviada para análisis morfológico e inmunohistoquimico. La inmunohistoquimica dio negatividad para actina, CD117, DOG-1 y Melan $A$, sin embargo, demostró reactividad para S100 y GFAP, confirmando el origen nervioso del tejido (Figura 3a). El estudio histológico mostró lla presencia de cuerpos de Verocay (Figura 3b). Ante estos hallazgos, se realizó el diagnóstico definitivo de un Schwannoma de colon.

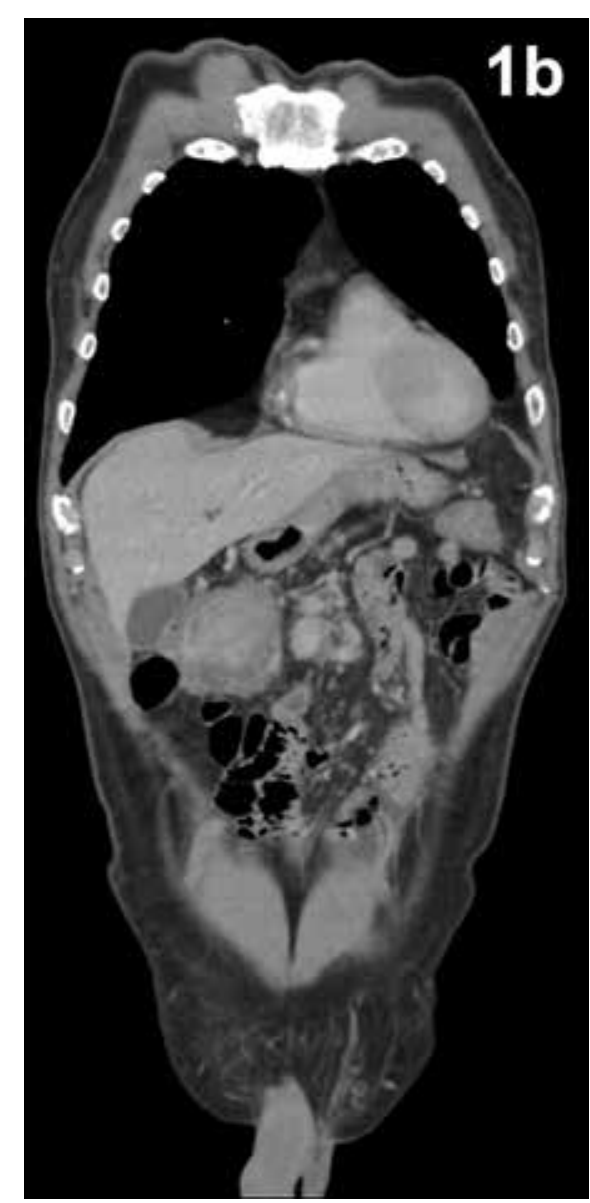

Figura 1: Tomografía computada de abdomen y pelvis en corte axial (a) y coronal (b). Se identifica una masa de aspecto neoplásico en el ángulo hepático, asociado a un segmento corto invaginado y marcados cambios inflamatorios de la grasa adyacente. 

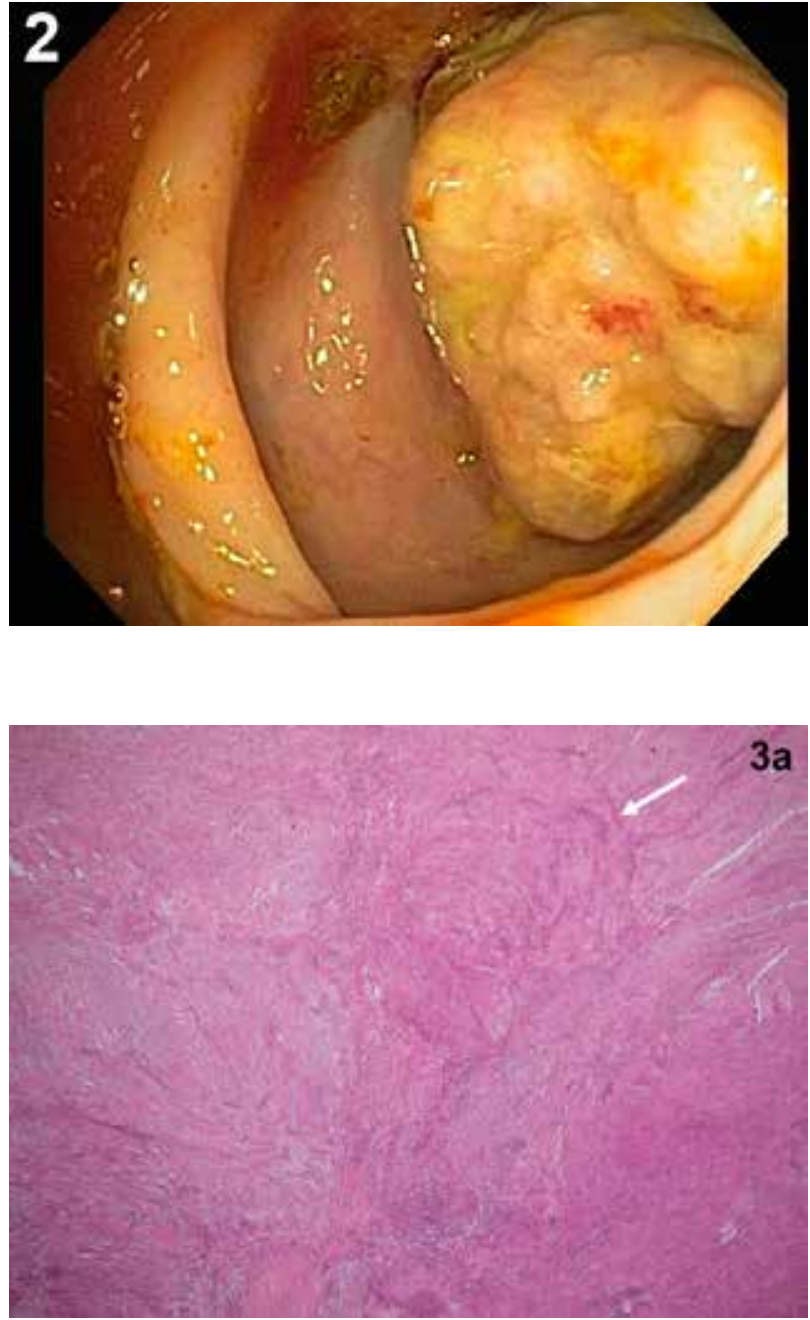

Figura 2: La colonoscopia evidencia la presencia de una lesión polipoidea de aspecto neoplásico, de consistencia dura y friable al tacto.

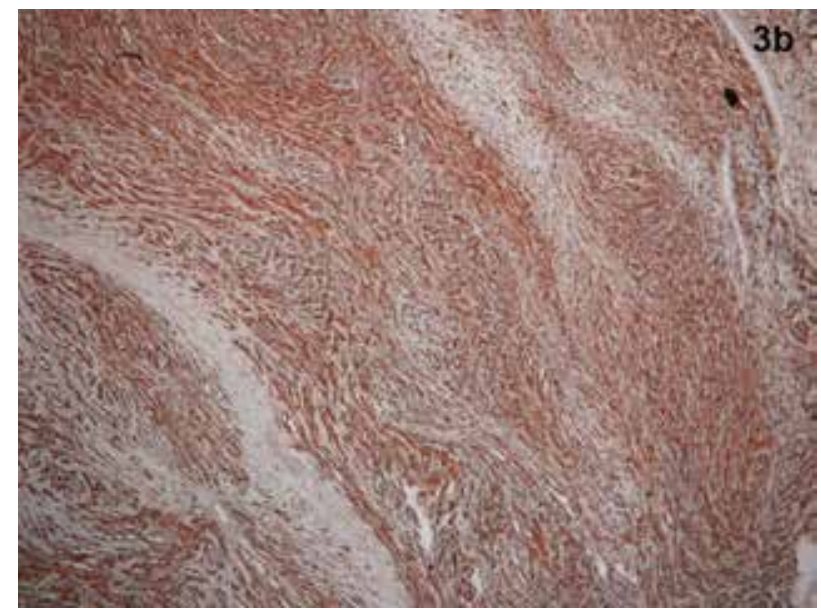

Figura 3: (a) Análisis histológico objetiva la presencia de cuerpos de Verocay que corresponden a una disposición apilada en empalizada de dos filas de núcleos alargados que alternan con zonas acelulares compuestas por procesos citoplasmáticos (Flecha blanca). (b) Análisis inmunihistoquímico muestra reactividad para S100 y GFAP, documentando la presencia de tejido nervioso.

\section{Discusión}

Aunque el número de informes de schwannomas gastrointestinales ha aumentado con los avances en la tinción inmunohistoquímica, siguen siendo tumores poco frecuentes. Los de ubicación colónica son bastante raros y corresponden a un $6,1 \%$ de todos los schwannomas gastrointestinales reportados hasta ahora en estudios previos. No hay predilección de género en la incidencia de SC y aparecen más comúnmente después de la sexta década de vida ${ }^{9}$. Generalmente se presenta como una masa que protruye desde la muscular propia donde se puede observar infiltración del musculo liso sin formar una verdadera cápsula.
Estas características fueron consideradas por algunos autores para reflejar el origen aparente del tumor en células de Schwann dispersas en la mucosa intestinal. Las imágenes preoperatorias son útiles para definir la ubicación exacta del tumor, sin embargo, no pueden determinar la benignidad o malignidad del tumor y existe una superposición entre las características de los SC y otros tumores submucosos. En la tomografía computada, los SC suelen aparecer como masas murales redondas, bien definidas, con realce homogéneo y sin evidencia de cambios quísticos, necrosis, hemorragia o calcificaciones ${ }^{10}$. El principal diagnóstico diferencial a considerar corresponde a 
los tumores estromales gastrointestinales (GIST), los cuales también presentan un origen submucoso y un comportamiento en imágenes variable según su tamaño y grado de malignidad. Las lesiones menores de $3 \mathrm{~cm}$ presentan un realce homogéneo, mientras que las lesiones de mayor tamaño demuestran áreas de necrosis con realce heterogéneo. Una característica imagenológica que ayuda a diferenciar los GIST de los SC, es que estos últimos, a pesar del tamaño, presentarán realce homogéneo sin áreas de necrosis y en las fases arteriales los SC pueden presentar ningún o leve realce periférico. Los adenocarcinomas de colon corresponden a otro diagnóstico diferencial, este se identifica como un engrosamiento parietal con densidad de partes blandas que pueden presentar áreas de ulceración y generar estenosis luminal ${ }^{11}$.

La biopsia endoscópica incisional suele ser insuficiente ya que esta lesión se ubica en la submucosa. El estándar de referencia es la resección total de la lesión para la evaluación histológica y la realización de estudio inmunohistoquímico ${ }^{12}$. El estudio inmunohistoquímico es de gran utilidad en el diagnóstico diferencial con otras lesiones como los GIST y los GANT (variante de los GIST derivados de los nervios autónomos), leiomiomas o leiomiosarcomas que pueden presentar un comportamiento más agresivo ${ }^{13}$. El schwannoma suele expresar reacción positiva para S-100, vimentina y GFAP, sin reactividad para el receptor transmembrana de tirosinaquinasa denominado C-KIT o CD117, el antígeno de células precursoras del sistema hematopoyético CD34, actina o citoqueratinas, que, sí se presentan más típicamente en los GIST, GANT o tumores musculares ${ }^{14}$. El tratamiento definitivo es la extirpación quirúrgica completa con márgenes libres de compromiso neoplásico. El uso de radioterapia o quimioterapia adyuvante tiene resultados contradictorios y no se recomienda rutinariamente. Estos pacientes presentan buen pronóstico dada su benignidad y es muy rara la recidiva o la presencia de metástasis ${ }^{15}$.

En conclusión, el SC es un tumor submucoso de colon infrecuente y la tomografía computada nos ayuda a localizarlo, identificar su extensión y planificar la cirugía. Sin embargo, son las técnicas de inmunohistoquímicas las cuales nos permiten confirmar el diagnóstico de un SC.

\section{Referencias}

1. Nonose R, Lahan AY, Santos Valenciano J, Martínez CA. Schwannoma of the Colon. Case Rep Gastroenterol 2009; 3: 293-299.

2. Miettinen M, Shekitka KM, Sobin LH. Schwannomas in the colon and rectum: A clinicopathologic and immu- nohistochemical study of 20 cases. Am J Surg Pathol 2001; 25: 846-855.

3. Kwon MS, Lee SS, Ahn GH. Schwannomas of the gastrointestinal tract: Clinicopathological features of 12 cases including a case of esophageal tumor compared with those of gastrointestinal stromal tumors and leiomyomas of the gastrointestinal tract. Pathol Res Pract. 2002; 198: 605-613.

4. Tanaka T, Ishihara Y, Takabayashi N, Kobayashi R, Hiramatsu T, Kuriki K. Gastrointestinal: Asymptomatic colonic schwannoma in an elderly woman; a rare case. J Gastroenterol Hepatol. 2011; 26: 1339.

5. Levy AD, Quiles AM, Miettinen M, Sobin LH. Gastrointestinal schwannomas: $C T$ features with clinicopathologic correlation. AJR Am J Roentgenol. 2005; 184: 797-802.

6. Yoon W, Paulson K, Mazzara P, Nagori S, Barawi M, Berri R. Gastric schwannoma: A rare but important differential diagnosis of a gastric submucosal mass. Case Rep Surg. 2012; 2012: 280982.

7. Hsu KF, Lin CT, Wu CC, Hsiao CW, Lee TY, Mai CM, et al. Schwan- noma of the rectum: report of a case and review of the literature. Rev Esp Enferm Dig 2010; 102: 289-291.

8. Fletcher CD, Berman JJ, Corless C, Gorstein F, Lasota $J$, Longley BJ, Miettinen M, O'Leary TJ, Remotti H, Rubin BP, Shmookler B, Sobin LH, Weiss SW. Diagnosis of gastrointestinal stromal tumors: a consensus approach. Int J Surg Pathol. 2002; 10: 81-89.

9. Prévot S, Bienvenu L, Vaillant JC, de Saint-Maur PP. Benign schwannoma of the digestive tract: a clinicopathologic and immunohistochemical study of five cases, including a case of esophageal tumor. $\mathrm{Am} \mathrm{J}$ Surg Pathol. 1999; 23: 431-436.

10. Levy AD, Quiles AM, Miettinen M, Sobin LH. Gastrointestinal schwannomas: CT features with clinicopathologic correlation. AJR Am J Roentgenol. 2005; 184: 797-802.

11. Kyung Lee N, et al. Hypervascular Subepithelial Gastrointestinal Masses: CT-Pathologic Correlation. RadioGraphics 2010; 30: 1915-1934.

12. Tedeschi M, Cuccia F, Angarano E, Piscitelli D, Gigante G, Altomare DF. Solitary schwannoma of the rectum mimicking rectal cancer. Ann Ital Chir. 2011; 82: 309312.

13. Tanoue $\mathrm{Y}$, Tanaka N, Nagai M, Suzuki Y. Benign pigmented schwannoma of the great omentum: Report of a rare case and review of the literature. Case Rep Gastroenterol. 2009; 3: 222-229.

14. Hou YY, Tan YS, Xu JF, Wang XN, Lu SH, Ji Y, Wang J, Zhu XZ. Schwannoma of the gastrointestinal tract: A clinicopathological, immunohistochemical and ultrastructural study of 33 cases. Histopathology. 2006; 48: 536-545.

15. Watanabe A, Ojima H, Suzuki S, Mochida Y, Hirayama I, Hosouchi $Y$, et al. An individual with gastric schwannoma with pathologically malignant potential surviving two years after laparoscopyassisted partial gastrectomy. Case Rep Gastroenterol. 2011; 5: 502-507. 